\title{
Disappearance of Meissner Effect and Specific Heat Jump in a Multiband Superconductor, $\mathrm{Ba}_{0.2} \mathrm{~K}_{0.8} \mathrm{Fe}_{2} \mathrm{As}_{2}$
}

\author{
Y. Tanaka $\cdot$ P.M. Shirage $\cdot$ A. Iyo
}

Received: 24 July 2009 / Accepted: 11 August 2009 / Published online: 9 October 2009

(C) The Author(s) 2009. This article is published with open access at Springerlink.com

\begin{abstract}
We observed the disappearance of the Meissner effect and the specific heat jump in $\mathrm{Ba}_{0.2} \mathrm{~K}_{0.8} \mathrm{Fe}_{2} \mathrm{As}_{2}$. A crossover of the type of superconducting order parameter can be a source of these phenomena. A quantum phase disorder provides a possible explanation.
\end{abstract}

Keywords Two-band superconductor · Two-component superconductor - Two-component quantum condensation . Soliton $\cdot$ i-soliton $\cdot$ Meissner effect $\cdot$ Specific heat .

Fe-based superconductor $\cdot \mathrm{SU}(2)$ superconductivity

\section{Introduction}

Two-component superconductivity observed in a two-band superconductor has a relative phase fluctuation between two bands (Leggett mode) [1]. Its rotation of $2 \pi$ results in a soliton (intercomponent phase difference soliton) [2], which makes a phase texture [3, 4]. These hierarchical structures exhibit rich physical phenomena and potential applications [5], which some researchers are seeking in the two-band superconductor [5-9].

An iron-based superconductor is one of the potential candidates $[10,11]$. Fermi surfaces can be classified into two groups: two electron Fermi surfaces and three hole Fermi surfaces [12-15]. We consider that these two groups comprise the two-band superconductor (two-component superconductor).

Y. Tanaka $(\bowtie) \cdot$ P.M. Shirage · A. Iyo

National Institute of Advanced Industrial Science and

Technology, Tsukuba 305-8568, Japan

e-mail: y.tanaka@aist.go.jp
An inverse isotope effect reported by Shirage et al. [16] suggests that there are two different interband pairing interactions, which are due to phononic and electronic ones. Because the electronic mediator reverses the sign of the order parameter between two Fermi surfaces whereas phononic one does not, we can tune the interband interaction strength. Yanagisawa et al. pointed out that it is possible to diminish the interband interaction by doping [17]. He called this abnormal situation "crossover."

In this report, we present the trial abnormal phenomena relating to this crossover.

\section{Experimental}

Polycrystalline samples of $\mathrm{Ba}_{0.6} \mathrm{~K}_{0.4} \mathrm{Fe}_{2} \mathrm{As}_{2}$ and $\mathrm{Ba}_{0.2} \mathrm{~K}_{0.8} \mathrm{Fe}_{2} \mathrm{As}_{2}$ are prepared by high-pressure synthesis [16]. The magnetization was measured using a Quantum Design Magnetic Property Measurement System (MPMS). The heat capacity was measured using a Quantum Design Physical Properties Measurement System (PPMS). These are typical measurements described in the previous reports $[16,18,19]$. The magnetizations are measured before and after the specific heat measurement of $\mathrm{Ba}_{0.2} \mathrm{~K}_{0.8} \mathrm{Fe}_{2} \mathrm{As}_{2}$ to confirm that there is no degradation of the superconducting property. The possible influence of the remnant field on the specific heat measurement was examined by comparing it with the data taken under $a<1$ Oe field, which was tuned at $300 \mathrm{~K}$ using a gauss-meter.

\section{Results}

The measured samples are indicated in Fig. 1 with $T_{\mathrm{c}}$ of $\mathrm{Ba}_{x} \mathrm{~K}_{1-x} \mathrm{Fe}_{2} \mathrm{As}_{2}$ we synthesized. 


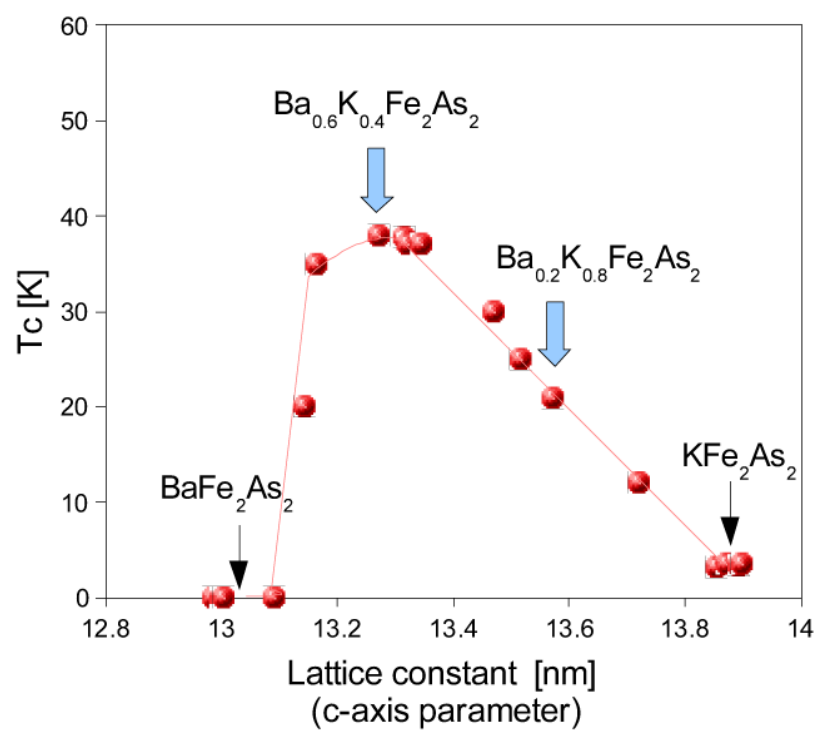

Fig. $1 T_{\mathrm{c}}$ of $\mathrm{Ba}_{x} \mathrm{~K}_{1-x} \mathrm{Fe}_{2} \mathrm{As}_{2}$ we synthesized. The samples we measured indicated by the bold arrows

Figure 2 shows the magnetic properties obtained using a zero-field-cooling process (ZFC) and field-cooling process (FC). The demagnetization due to the shielding current gives almost $100 \%$ volume fraction. $\mathrm{Ba}_{0.6} \mathrm{~K}_{0.4} \mathrm{Fe}_{2} \mathrm{As}_{2}$ gave a definite Meissner signal in $\mathrm{FC}$, while $\mathrm{Ba}_{0.2} \mathrm{~K}_{0.8} \mathrm{Fe}_{2} \mathrm{As}_{2}$ did not.

The superconducting transition temperatures $\left(T_{\mathrm{c}}\right)$ determined by the ZFC signal are 38 and $21 \mathrm{~K}$ for $\mathrm{Ba}_{0.6} \mathrm{~K}_{0.4} \mathrm{Fe}_{2} \mathrm{As}_{2}$ and $\mathrm{Ba}_{0.2} \mathrm{~K}_{0.8} \mathrm{Fe}_{2} \mathrm{As}_{2}$, respectively. The transition is very clear and there is no meaningful broadening.

Figure 3 shows the heat capacity. A clear jump was observed at $T_{\mathrm{c}}$ in $\mathrm{Ba}_{0.6} \mathrm{~K}_{0.4} \mathrm{Fe}_{2} \mathrm{As}_{2}$. It is consistent with a previous report [20, 21]. However, the jump cannot be seen in $\mathrm{Ba}_{0.2} \mathrm{~K}_{0.8} \mathrm{Fe}_{2} \mathrm{As}_{2}$.

\section{Discussion}

Disappearance of the specific heat jump has been reported in under-doped cuprate superconductor [22]. Loram et al. explained that a BCS pair forms well above $T_{\mathrm{c}}$ and a large portion of the entropy is already lost at $T_{\mathrm{c}}$ [23].

When there are large residual un-paired carriers, the height of the jump is also reduced.

In both cases, we expect the condensation energy of the superconductivity to decrease. This weakens the pinning potential of the vortex.

The lack of a Meissner signal can be expected when there is strong pinning. Superficially, we can consider the lack of a Meissner signal in $\mathrm{Ba}_{0.2} \mathrm{~K}_{0.8} \mathrm{Fe}_{2} \mathrm{As}_{2}$ to be due to a large pinning potential. When $\mathrm{Ba}$ and $\mathrm{K}$ disorder creates a pinning potential, we would expect also strong pinning in $\mathrm{Ba}_{0.6} \mathrm{~K}_{0.4} \mathrm{Fe}_{2} \mathrm{As}_{2}$. But $\mathrm{Ba}_{0.6} \mathrm{~K}_{0.4} \mathrm{Fe}_{2} \mathrm{As}_{2}$ gives the usual Meissner signal. We cannot find any mechanism to give a large pinning potential from a material science viewpoint.

The lack of specific heat jump is not consistent with the presence of a strong pinning center either.

When there is no Meissner signal, we also expect that superconductivity happens only at the surface. A robust ZFC signal is rather unlikely in this case.

Fukazawa et al. reported a clear specific heat jump in $\mathrm{KFe}_{2} \mathrm{As}_{2}$, the last member of this system [24]. This sample also gives the Meissner signal. $\mathrm{Ba}_{0.6} \mathrm{~K}_{0.4} \mathrm{Fe}_{2} \mathrm{As}_{2}$ has the highest $T_{\mathrm{c}}$ and $\mathrm{KFe}_{2} \mathrm{As}_{2}$ is the last member. Both give a definite jump in the specific heat and the Meissner signal. Only an in-between member, $\mathrm{Ba}_{0.2} \mathrm{~K}_{0.8} \mathrm{Fe}_{2} \mathrm{As}_{2}$, lacks both of the jump and the Meissner signal.

Yanagisawa et al. theoretically predicted the abnormal superconductivity for the in-between member [17]. The scenario is based on a two-band superconductivity. In this scenario, the sign of the order parameters for two-band is the same for one end member $(++$ wave), while it is reversed in another member ( +- wave). In the in-between member, the ++ wave and the +- wave compete. It can be considered to be a weakly-coupled two-order-parameter superconductivity. (Of course, the competition between the other types of order parameters, including d-wave, qualitatively leads to the same physical consequence, which will be described below.).

The soliton, in which the relative phase between two order parameters rotates $2 \pi$, can emerge $[3,4,7]$ when there are two order parameters (or two components). Owing to the phase difference soliton, the quantum phase texture tends to be invoked for the film sample [3, 7, 9]. The small grains can also satisfy the necessary criterion.

This texture potentially leads to disappearance of the Meissner signal and the specific heat jump. Figure 4 illustrates these situations. According to Goryo, the phase difference soliton is thermally activated just below $T_{\mathrm{c}}$ [7]. This mechanism can work regardless of whether there is a vortex [9]. Just below $T_{\mathrm{c}}$, there is a quantum phase texture. This texture moves and causes energy loss. Neither ZFC nor FC signals are to be expected. With decreasing temperature, the motion of the soliton walls slows down. The system goes into a quantum phase glassy state. This is similar to glass transition. No specific heat jump is to be expected. It should be noted that the phase difference soliton is inactive against the electromagnetic field. Therefore, this phase texture cannot be eliminated by the real current flow. The geometry of the grain (there are many hollows, bottlenecks and surface roughness in the sample) becomes the effective pinning center for the solitons.

When there is a magnetic field, the vortex will be trapped by the soliton walls and kept inside the grain. This inhibits ejection of the vortex and the Meissner effect. 


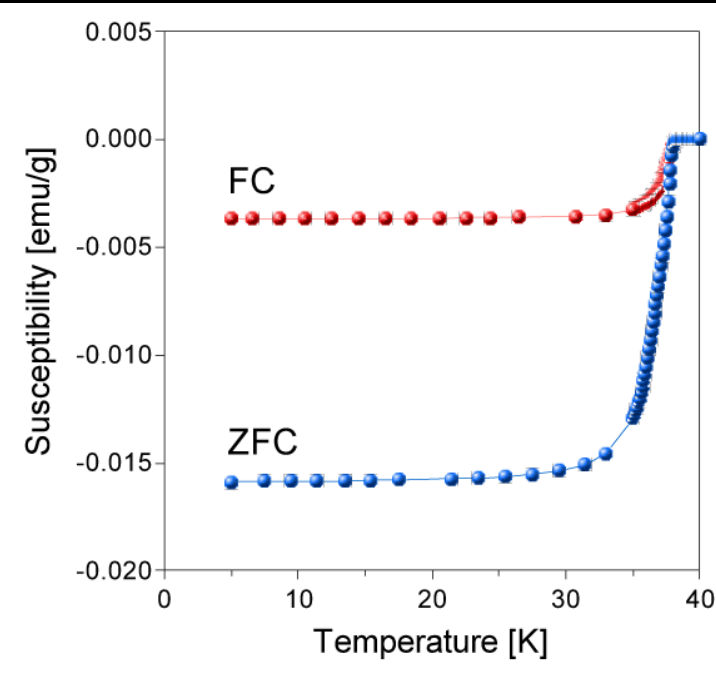

(a)

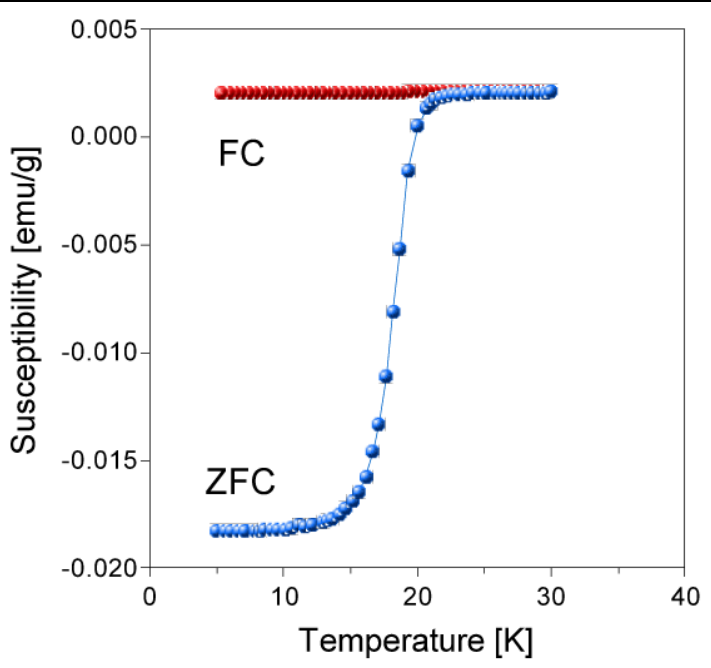

(b)

Fig. 2 Magnetic properties of (a) $\mathrm{Ba}_{0.6} \mathrm{~K}_{0.4} \mathrm{Fe}_{2} \mathrm{As}_{2}$ and (b) $\mathrm{Ba}_{0.2} \mathrm{~K}_{0.8} \mathrm{Fe}_{2} \mathrm{As}_{2}$. No Meissner signal was observed in $\mathrm{Ba}_{0.2} \mathrm{~K}_{0.8} \mathrm{Fe}_{2} \mathrm{As}_{2}$

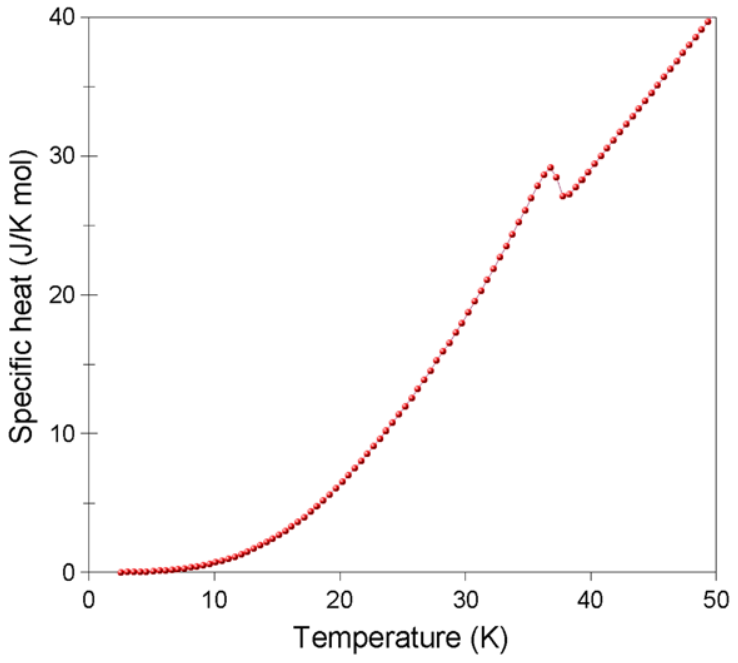

(a)

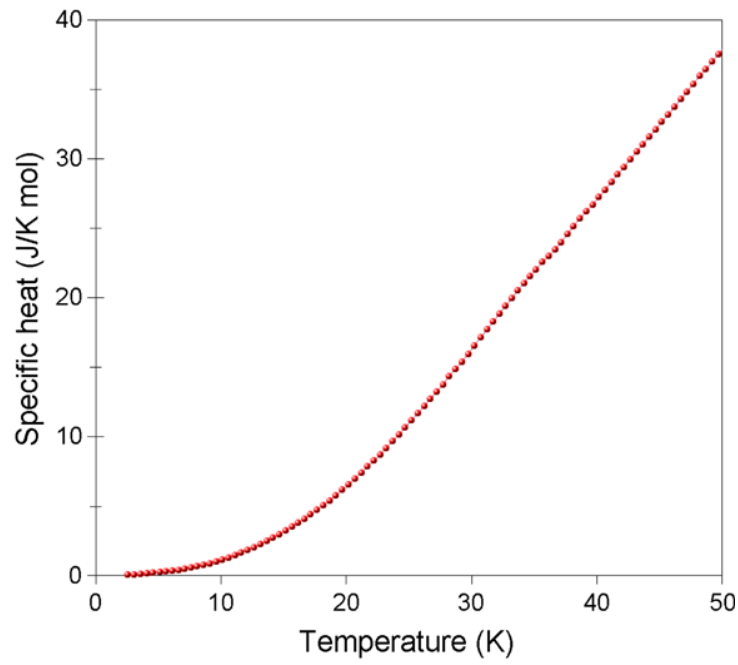

(b)

Fig. 3 Specific heat of (a) $\mathrm{Ba}_{0.6} \mathrm{~K}_{0.4} \mathrm{Fe}_{2} \mathrm{As}_{2}$ and (b) $\mathrm{Ba}_{0.2} \mathrm{~K}_{0.8} \mathrm{Fe}_{2} \mathrm{As}_{2}$ without magnetic field. No specific heat jump was observed in $\mathrm{Ba}_{0.2} \mathrm{~K}_{0.8} \mathrm{Fe}_{2} \mathrm{As}_{2}$

A similar texture structure and its dynamics have also been reported in other multicomponent systems such as the superfluid ${ }^{3} \mathrm{He}$ [25-29]. We can expect a similar situation to occur in $\mathrm{Ba}_{0.2} \mathrm{~K}_{0.8} \mathrm{Fe}_{2} \mathrm{As}_{2}$.

When two order parameters are almost degenerated, the soliton wall becomes thick and converts into the domain itself [30]. It is similar to the chiral domain structure of the triplet superconductors [31-34] or the texture in twocomponent Bose-Einstein condensates [35]. In this situation, the system enters into an ultimate quantum disorder phase. This disorder is easily triggered by an inhomogeneity of the local crystal structures. Moreover, this domain wall can trap many fractional vortices [31, 32].
Of course, the explanation is still tentative and the experimental study is still to be undertaken. But this possibility will be taken into account in future study.

\section{Summary}

The two-band superconductivity realized in the iron-based superconductor can be considered as the abnormal quantum condensation like the superfluid ${ }^{3} \mathrm{He}$. The disappearance of the Meissner effect and the specific heat jump possibly reflect this abnormal situation. 


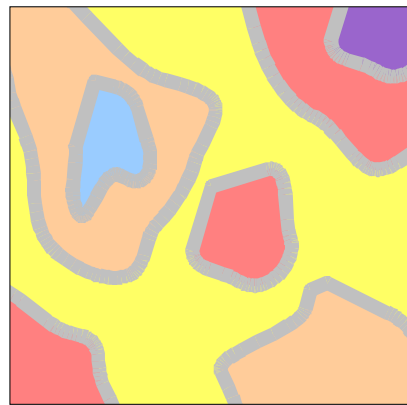

(a)

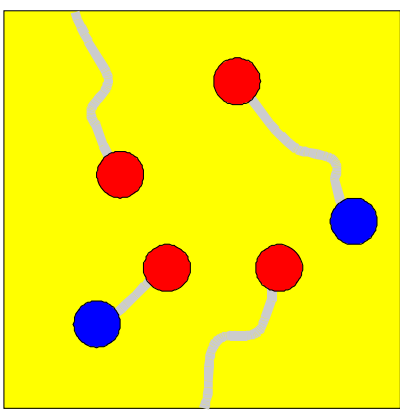

(b)
Fig. 4 (Color online) Schematic diagram of the quantum phase texture. The solid gray lines indicate soliton walls. The different quantum phases of the phase domain surrounded by the soliton wall are indicated by different colors. The large red and blue points indicate fractional vortices. There are two fractional vortices, each having different quantum flux quanta. Soliton walls are generated just below $T_{\mathrm{c}}$ and are moving. (a) With decreasing temperature, they gradually stop. This is the quantum glassy state. When there is a magnetic field, the fractional vortex is trapped by a soliton and the soliton wall is terminated by the fractional vortex (b)

Acknowledgements This work was supported by the Strategic International Cooperative Program of Japan Science and Technology Agency (JST) and the Department of Science and Technology (India) "Feasibility Study of the Application of Multiple-order Parameters in Materials to Information Processing", and a Grant-in-Aid for Specially promoted Research (20001004) from The Ministry of Education, Culture, Sports, Science and Technology (MEXT) and the AIST through a grant for overriding priority research "New Superconducting Revolution".

Open Access This article is distributed under the terms of the Creative Commons Attribution Noncommercial License which permits any noncommercial use, distribution, and reproduction in any medium, provided the original author(s) and source are credited.

\section{References}

1. Leggett, A.J.: Prog. Theor. Phys. 36, 901 (1966)

2. Tanaka, Y.: J. Phys. Soc. Jpn. 70, 2844 (2001)

3. Gurevich, A., Vinokur, V.M.: Phys. Rev. Lett. 90, 047004 (2003)

4. Tanaka, Y.: Phys. Rev. Lett. 88, 017002 (2002)

5. Gurevich, A., Vinokur, V.M.: Phys. Rev. Lett. 97, 137003 (2006)

6. Bluhm, H., Koshnick, N.C., Huber, M.E., Moler, K.A.: Phys. Rev. Lett. 97, 237002 (2006). (See also erratum, Phys. Rev. Lett. 98, 209902 (2007) (E))

7. Goryo, J., Soma, S., Matsukawa, H.: Europhys. Lett. 80, 17002 (2007)

8. Crisan, A., Tanaka, Y., Shivagan, D.D., Iyo, A., Cosereanu, L., Tokiwa, K., Watanabe, T.: Jpn. J. Appl. Phys. 46, L451 (2007)
9. Tanaka, Y., Crisan, A., Shivagan, D.D., Iyo, A., Tokiwa, K., Watanabe, T.: Jpn. J. Appl. Phys. 46, 134 (2007) and references therein

10. Kamihara, Y., Hiramatsu, H., Hirabo, M., Kawamura, R., Yanagi, H., Kamiya, T., Hosono, H.: J. Am. Chem. Soc. (Communication) 128(2006), 10012-10013 (2006)

11. Rotter, M., Tegel, M., Schellenberg, I., Hermes, W., Pottgen, R., Johrendt, D.: Phys. Rev. B 78, 020503(R) (2008)

12. Kuroki, K., Onari, S., Arita, R., Usui, H., Tanaka, Y., Kontani, H., Aoki, H.: Phys. Rev. Lett. 101, 087004 (2008)

13. Mazin, I.I., Singh, D.J., Johannes, M.D., Du, M.H.: Phys. Rev. Lett. 101, 057003 (2008)

14. Fukuda, T., Baron, A.Q.R., Shamoto, S.I., Ishikado, M., Nakamura, H., Machida, M., Uchiyama, H., Tsutsui, S., Iyo, A., Kito, H., Arai, J.M.M., Eisaki, H., Hosono, H.: J. Phys. Soc. Jpn. 77, $103715(2008)$

15. Nekrasov, I.A., Pchelkina, Z.V., Sadovskii, M.V.: JETP Lett. 88, 679 (2008)

16. Shirage, P.M., Kihou, K., Miyazawa, K., Lee, C.H., Kito, H., Eisaki, H., Tanaka, Y., Iyo, A.: Preprint arXiv:0903.3515 (2009)

17. Yanagisawa, T., Odagiri, K., Hase, I., Yamajia, K., Shiragea, P.M., Tanakaa, Y., Iyo, A., Eisakia, H.: J. Phys. Soc. Jpn. 78, 094718 (2009)

18. Tanaka, Y., Iyo, A., Shirakawa, N., Ariyama, M., Tokumoto, M., Ikeda, S.I., Ihara, H.: J. Phys. Soc. Jpn. 70, 329-332 (2001)

19. Tanaka, Y., Iyo, A., Shirakawa, N., Ariyama, M., Tokumoto, M., Ikeda, S.I., Ihara, H.: Physica C 357, 222 (2001)

20. Luo, G.M.H., Wang, Z., Shan, L., Ren, C., Wen, H.-H.: Preprint arXiv:0808.2941 (2008)

21. Welp, U., Xie, R., Koshelev, A.E., Kwok, W.K., Luo, H.Q., Wang, Z.S., Mu, G., Wen, H.H.: Preprint arXiv:0810.1944 (2008)

22. Loram, J.M., Mirza, K.A., Cooper, J.R., Liang, W.Y.: Phys. Rev. Lett. 71, 1740 (1993)

23. Loram, J.M., Mirza, K.A., Cooper, J.R., Liang, W.Y., Wade, J.M.: J. Supercond. 7, 243 (1994)

24. Fukazawa, H., Yamada, Y., Kondo, K., Saito, T., Kohori, Y., Kuga, K., Matsumoto, Y., Nakatsuji, S., Kito, H., Shirage, P.M., Kihou, K., Takeshita, N., Lee, C.H., Iyo, A., Eisaki, H.: Preprint arXiv:0906.4644 (2009)

25. Ruutu, V.M., Parts, U., Krusius, N.: J. Low. Temp. Phys. 103, 331 (1996)

26. Thuneberg, E.V.: J. Low. Temp. Phys. 101, 135 (1995)

27. Part, U., Thuneberg, E.V., Volovik, G.E., Koivuniemi, J.H., Ruutu, V.M.H., Heinila, M., Karimaki, J.M., Krusius, M.: Phys. Rev. Lett. 72, 3839 (1994)

28. Korhonen, J.S., Kondo, Y., Krusius, M., Thuneberg, E.V., Volovik, G.E.: Phys. Rev. B. 47, 8868 (1993)

29. Kondo, Y., Korhonen, J.S., Krusius, M., Dmitriev, V.V., Mukharsky, Y.M., Sonin, E.B., Volovik, G.E.: Phys. Rev. Lett. 67, $81(1991)$

30. Tanaka, Y., Shirage, P.M., Iyo, A.: Submitted to Physica B

31. Sigrist, M., Agterberg, D.F.: Prog. Theor. Phys. 102, 965 (1999)

32. Izyumov, Yu.A., Laptev, V.M.: Phase Transition 20, 95 (1990)

33. Matsunaga, Y., Ichioka, M., Machida, K.: Phys. Rev. B 70, 10052 (2004) (R)

34. Matsunaga, Y., Ichioka, M., Machida, K.: Phys. Rev. Lett. 92, 157001 (2004)

35. Kasamatsu, K., Tsubota, M.: Phys. Rev. A 79, 023606 (2009) 